\title{
Tumor Selective Silencing Using an RNAi-Conjugated Polymeric Nanopharmaceutical
}

\section{Supplementary Information}

Sonke Svenson, Roy I. Case, Roderick O. Cole, Jungyeon Hwang, Sujan R. Kabir, Douglas Lazarus, Patrick Lim Soo, Pei-Sze Ng, Christian Peters, Pochi Shum, Beata Sweryda-Krawiec, Snehlata Tripathi, Derek van der Poll, Scott Eliasof*

\section{Synthesis}

\subsection{Synthesis of 2-(2-(Pyridin-2-yl)disulfanyl)ethylamino-5050-PLGA-O-acetyl}

5050-PLGA-O-acetyl, 2 (2.0 g, $0.32 \mathrm{mmol})$, N-hydroxysuccinimide (66 mg, $0.57 \mathrm{mmol}$ ) and 1ethyl-3-(3-dimethylaminopropyl)carbodiimide (122 $\mathrm{mg}, 0.63 \mathrm{mmol})$ were dissolved in dimethylformamide (DMF, $12 \mathrm{~mL}$ ). To this reaction mixture, 2-(2-(pyridin-2yl)disulfanyl)ethylamine, 3 (127 mg, $0.57 \mathrm{mmol}$ ) and diisopropylethylamine ( $82 \mathrm{mg}, 0.63 \mathrm{mmol})$ in dichloromethane $(D C M, 6 \mathrm{~mL})$ were added. The reaction mixture was then stirred at room temperature for $4 \mathrm{~h}$. Water $(40 \mathrm{~mL})$ was added to the reaction mixture, the resulting rubbery solid dissolved in DCM $(15 \mathrm{~mL})$ and washed twice with $0.1 \%$ aqueous $\mathrm{HCl}$ solution $(50 \mathrm{~mL} \times 2)$, followed by brine $(100 \mathrm{~mL})$. The organic layer was dried over sodium sulfate and further purified by precipitation into cold ether $(100 \mathrm{~mL})$. The solvent was removed, and the residue was dried under vacuum to yield a white solid $(1.4 \mathrm{~g}, 68 \%$ yield).

\subsection{Synthesis of 5050-PLGA-O-acetyl-polylysine}

5050-PLGA-O-acetyl (5.0 g, $1.1 \mathrm{mmol}$ ) was dissolved in anhydrous dimethyl formamide (25 mL). $\mathrm{N}$-hydroxysuccinamide $(190 \mathrm{mg}, 1.7 \mathrm{mmol}$ ) and dicyclohexylcarbodiimide (350 mg, $1.7 \mathrm{mmol}$ ) was added to the reaction mixture and the reaction was stirred for $3 \mathrm{~h}$. Poly- $\varepsilon-\mathrm{Cbz}-\mathrm{L}-\mathrm{lysine}$ (Sigma; mw 500-4,000Da; $1.0 \mathrm{~g}, 1.1 \mathrm{mmol}$ ) and triethylamine (290 mg, $2.8 \mathrm{mmol}, 390 \mathrm{~mL}$ ) were added to the above reaction mixture and the reaction was stirred overnight at room temperature. The reaction mixture was filtered using a $0.3 \mu \mathrm{m}$ in-line filter to remove the byproduct dicyclohexyl urea (DCU) and the product was precipitated using $300 \mathrm{~mL}$ of methanol. The precipitated material was washed with diethyl ether $(2 x)$. The product was further 
dissolved in anhydrous dichloromethane $(30 \mathrm{~mL}$ ) followed by the addition of $30 \mathrm{~mL}$ of $33 \%$ hydrogen bromide $(\mathrm{HBr}) /$ acetic acid solution and stirred for $2 \mathrm{~h}$. The product was precipitated by adding $100 \mathrm{~mL}$ of diethyl ether and washed two times. Finally, the precipitated material was dissolved in RNase free water and lyophilized to obtain final product as fluffy powder (final product: $1.54 \mathrm{~g})$.

\subsection{Synthesis of Oligonucleotide-C6-5050-PLGA-O-acetyl Conjugate}

C6-Thiol modified oligonucleotides, 4 (siRNA, $20 \mathrm{mg}, 1.5 \mu \mathrm{mol}$, mw $13.2 \mathrm{kDa}$ ) was dissolved in

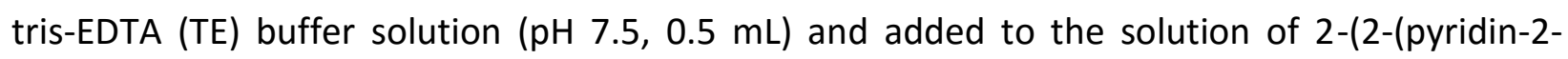
yl)disulfanyl)ethylamino-5050-PLGA-O-acetyl, 1 (85 mg, $12 \mu \mathrm{mol}$, mw 6.9 kD) in dimethyl sulfoxide (DMSO, $9.5 \mathrm{~mL}$ ), giving a DMSO:TE 95:5 v/v ratio, and incubated at $65^{\circ} \mathrm{C}$ for $3 \mathrm{~h}$. The reaction mixture was gradually cooled to room temperature and used for formulation without further purification. Conjugation efficiency of oligonucleotide-5050-PLGA-O-acetyl conjugate was analyzed by reverse phase HPLC, monitoring the amount of free pyridine-2-thione. The solution was kept frozen until needed for the formulation step. Both $\operatorname{siRNA}(G F P)$ and siRNA(AHA1) were conjugated with 2-(2-(pyridin-2-yl)disulfanyl)ethylamino-5050-PLGA-Oacetyl as described here. For dye-labeled siRNA strands, thiolated GFP sense single strand (10 mg; dissolved in 1xTE buffer) was mixed with the solution of 2-(2-(pyridin-2yl)disulfanyl)ethylamino-5050-PLGA-O-acetyl (85 mg, $12 \mu \mathrm{mol}, \mathrm{mw} 6.9$ kD) in dimethyl sulfoxide (DMSO, $9.5 \mathrm{~mL}$ ), giving a DMSO:TE 95:5 v/v ratio, and incubated at $65^{\circ} \mathrm{C}$ for $3 \mathrm{~h}$. The reaction mixture was cooled down to room temperature followed by addition of equimolar antisense DY647-labeled RNA strand and heating the resulting mixture to $95^{\circ} \mathrm{C}$ for annealing. After five minutes the reaction was cooled down gradually to room temperature. 


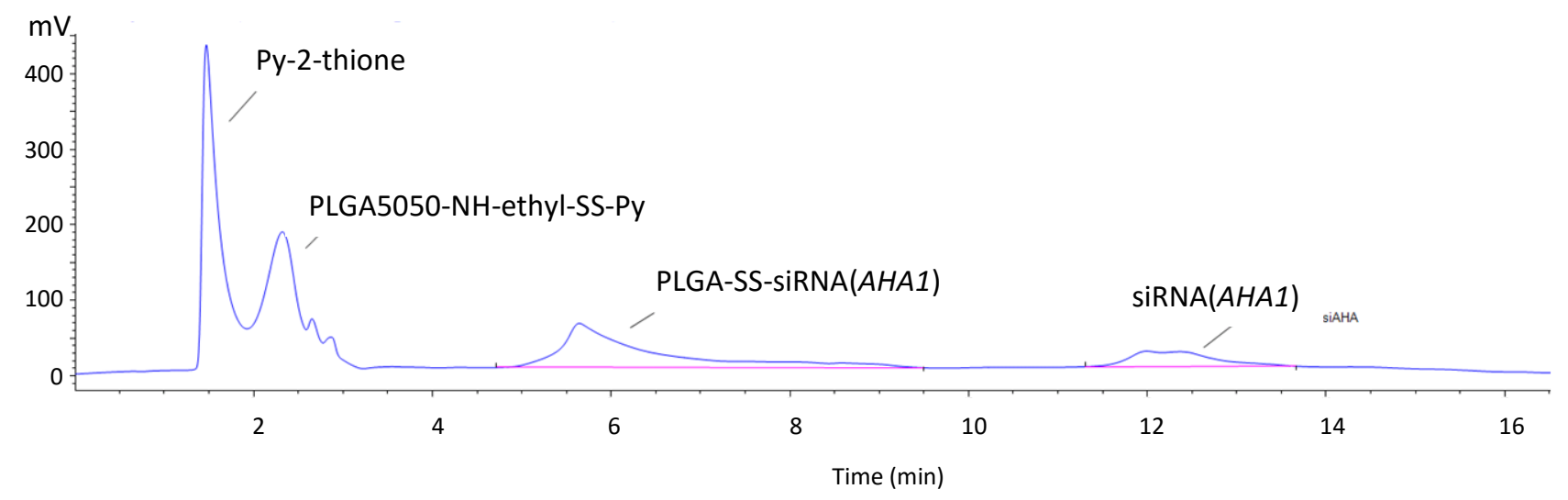

Supplementary Figure 1A. HPLC chromatogram separating PLGA-SS-siRNA(AHA1) from released activation group pyridine-2-thione, and unreacted PLGA5050-NH-ethyl-SS-Py as well as $\operatorname{siRNA}(A H A 1)-\mathrm{SH}$.

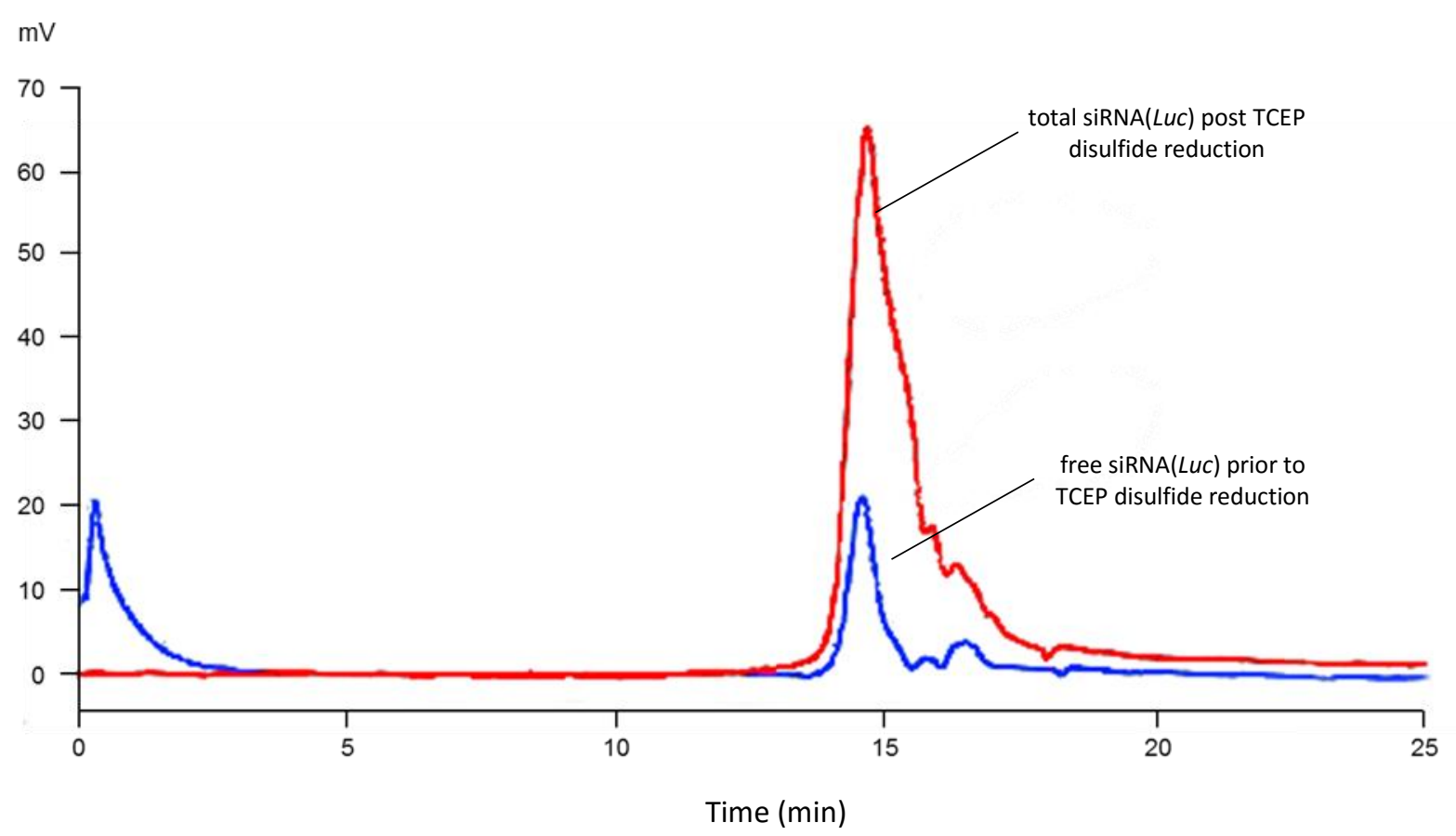

Supplementary Figure 1B. HPLC chromatogram showing the overlay of free and total SiRNA $(L U C)$ from formulations before and post treatment with reducing agent TCEP. The difference between total and free siRNA(LUC) gave the amount of conjugated siRNA(LUC). 

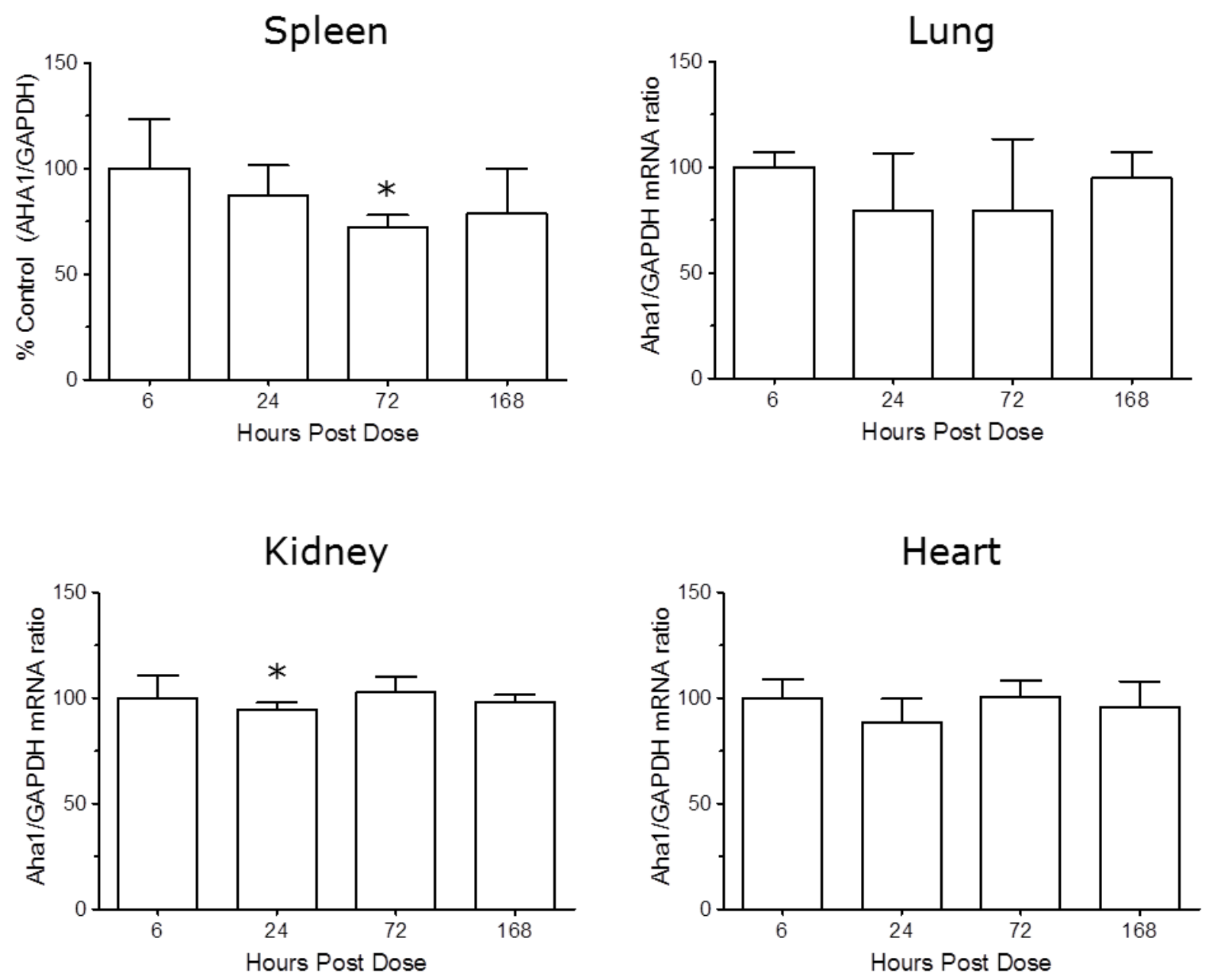

Supplementary Figure 2. Knockdown of mRNA(AHA1) at various time points in spleen, lung, kidney and heart following a single tail vein administration of $3 \mathrm{mg} / \mathrm{kg}$ siRNA(AHA1) PNPs in mice bearing HT-29 tumors (same experiment as Figure 3). Knockdown was normalized to mRNA(GAPDH) expression and is plotted as a percentage of the initial time point. Error bars represent standard deviation. ${ }^{*}=p<0.05$ relative to $100 \%$. 


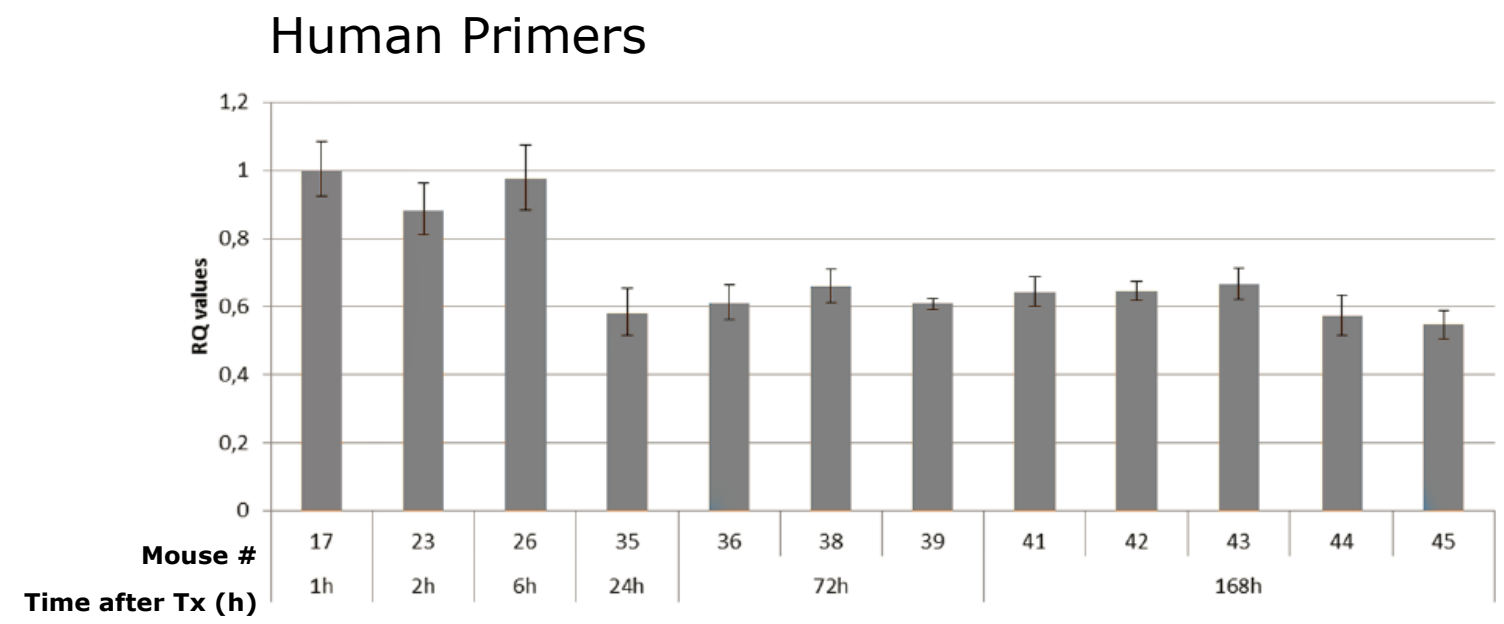

\section{Mouse Primers}

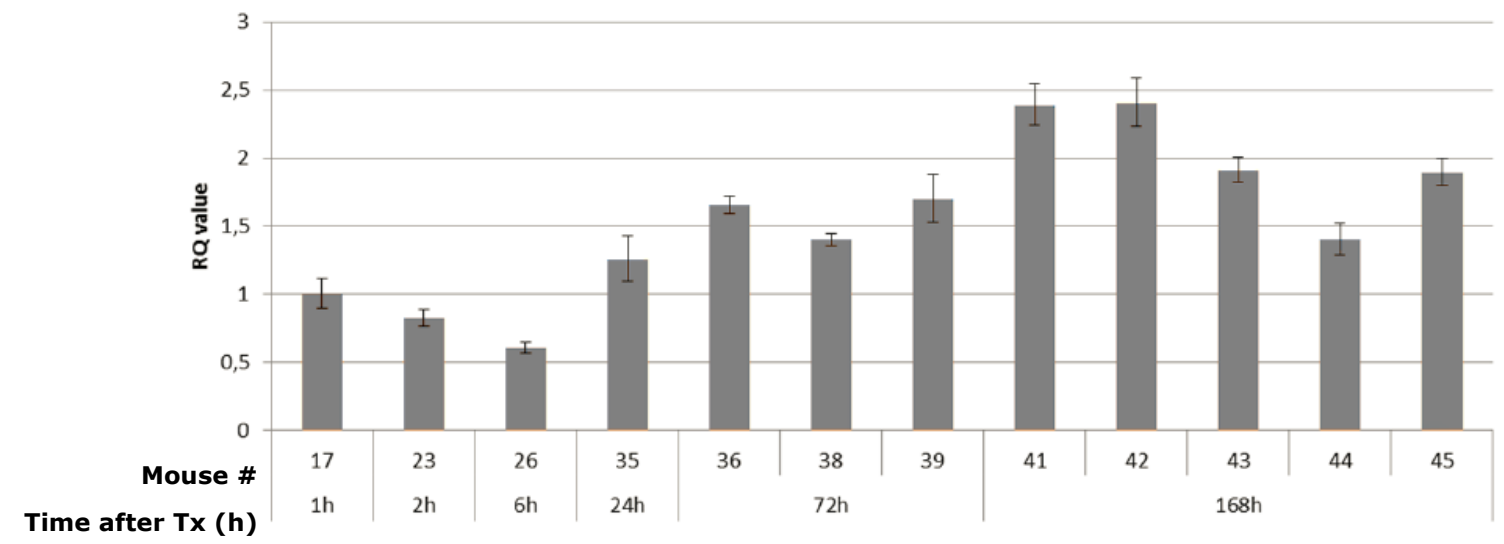

Supplementary Figure 3. Quantitative real-time PCR of $A H A 1$ in tumors from individual mice at various time points following a single tail vein administration of $3 \mathrm{mg} / \mathrm{kg}$ siRNA(AHA1) PNPs in mice bearing HT-29 tumors. Unlike Figure 3 and Supplementary Figure 1, primers were designed to be specific for either human or mouse mRNA(AHA1). Consistent knockdown was observed with human primers but not with mouse primers, suggesting that the knockdown of mRNA(AHA1) observed in these experiments was predominantly tumor AHA1. 
Vehicle-Treated Tumor

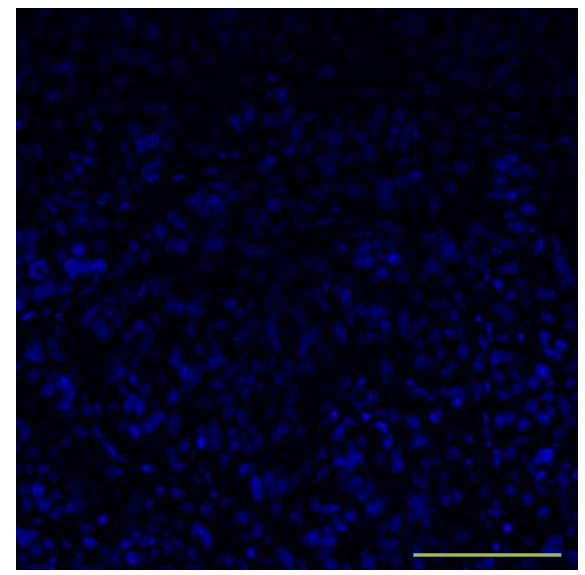

siRNA-PNP Treated Tumor

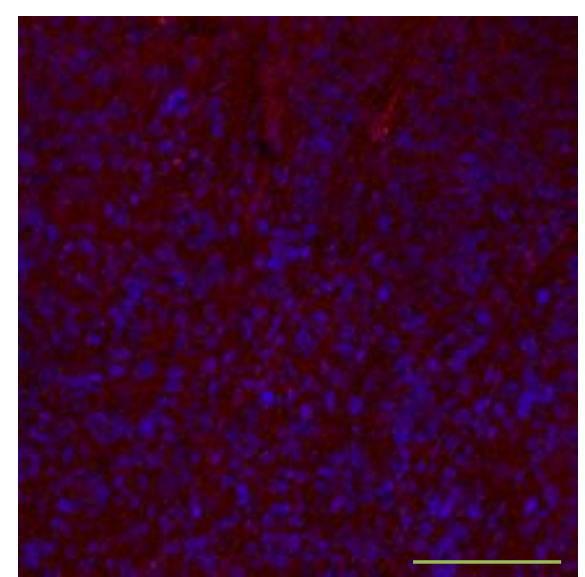

Supplementary Figure 4. Confocal micrographs demonstrating tumor localization of siRNA at various time points following a single tail vein administration of either vehicle (left) or $3 \mathrm{mg} / \mathrm{kg}$ dye-labeled (red) siRNA(GFP) PNPs (right) in mice bearing HT-29 tumors. Scale bar=100 $\mu \mathrm{m}$. 
Supplementary Table 1. Example of siRNA(LUC) loading measured by HPLC analysis and Quant-iT Ribogreen ${ }^{\circledR}$ reagent. Both methods gave identical values within experimental error.

\begin{tabular}{|c|c|c|c|}
\hline Formulation ID & $\begin{array}{c}\text { HPLC Conc } \\
(\mathrm{mg} / \mathrm{mL})\end{array}$ & $\begin{array}{c}\text { Ribogreen Conc } \\
(\mathrm{mg} / \mathrm{mL})\end{array}$ & $\begin{array}{c}\text { Ribogreen } \\
\text { Recovery (\%) }\end{array}$ \\
\hline $68-187 \mathrm{~A}$ & 1.28 & 1.30 & 101.9 \\
\hline $68-187 \mathrm{~B}$ & 0.99 & 1.03 & 104.3 \\
\hline
\end{tabular}

Supplementary Table 2. Body weight change and blood count $48 \mathrm{~h}$ following treatment with either vehicle (10\% sucrose) or siRNA PNPs administered at $11 \mathrm{mg} / \mathrm{kg}$ daily for 3 days. $* *=0.001<\mathrm{p}<0.01$

\begin{tabular}{|c|c|c|c|c|c|c|c|c|c|c|}
\hline Treatment & $\begin{array}{c}\text { Body Wt Change } \\
\% \text { Initial BWt }\end{array}$ & $\begin{array}{c}\text { WBC } \\
10^{3} / \mu \mathrm{L}\end{array}$ & $\begin{array}{c}\text { RBC } \\
10^{6} / \mu \mathrm{L}\end{array}$ & $\begin{array}{l}\text { HGB } \\
\mathrm{g} / \mathrm{dL}\end{array}$ & $\begin{array}{c}\text { HCT } \\
\% \\
\end{array}$ & $\begin{array}{c}\text { NEUTROPHIL } \\
\operatorname{per} \mu \mathrm{L}\end{array}$ & $\begin{array}{c}\text { LYMPHOCYTE } \\
\text { per } \mu \mathrm{L}\end{array}$ & $\begin{array}{c}\text { MONOCYTE } \\
\text { per } \mu \mathrm{L}\end{array}$ & $\begin{array}{c}\text { EOSINOPHIL } \\
\text { per } \mu \mathrm{L}\end{array}$ & $\begin{array}{c}\text { BASOPHIL } \\
\text { per } \mu \mathrm{L}\end{array}$ \\
\hline \multicolumn{11}{|l|}{ Vehicle } \\
\hline mean & 101.4 & 8.6 & 10.0 & 15.1 & 56.0 & 810.8 & 7331.6 & 59.0 & 223.8 & 42.4 \\
\hline SD & 1.6 & 1.1 & 0.3 & 0.4 & 1.3 & 140.4 & 916.1 & 13.3 & 59.8 & 24.5 \\
\hline \multicolumn{11}{|l|}{ siRNA PNP, 11 mg/kg } \\
\hline mean & 102.7 & 6.4 & 9.7 & 14.3 & 54.0 & 478.0 & 5645.4 & 49.4 & 120.0 & 19.4 \\
\hline SD & 1.8 & 2.1 & 0.1 & 0.3 & 1.0 & 125.4 & 1925.8 & 25.0 & 106.7 & 20.0 \\
\hline \multicolumn{2}{|r|}{ Reference range } & $5.2-9.2$ & $9.10-10.38$ & $14.4-16.0$ & $43.4-50.8$ & $780-2800$ & $3120-8960$ & $52-336$ & $0-672$ & $0-112$ \\
\hline $\begin{array}{l}\text { One-way ANOVA with Dunnett's } \\
\text { multiple comparison test }\end{array}$ & NS & NS & NS & $* *$ & NS & NS & NS & NS & NS & NS \\
\hline
\end{tabular}


Supplementary Table 3. Serum chemistry $48 \mathrm{~h}$ following treatment with either vehicle (10\% sucrose) or siRNA PNPs administered at $11 \mathrm{mg} / \mathrm{kg}$ daily for 3 days.

\begin{tabular}{|c|c|c|c|c|c|c|c|c|c|c|c|c|c|c|}
\hline Treatment & $\begin{array}{c}\text { ALK PHOSPHATASE } \\
\text { IU/L }\end{array}$ & $\begin{array}{l}\text { ALT } \\
\text { IU/L }\end{array}$ & $\begin{array}{l}\text { AST } \\
\mathrm{IU} / \mathrm{L}\end{array}$ & $\begin{array}{l}\mathrm{CPK} \\
\mathrm{IU} / \mathrm{L} \\
\end{array}$ & $\begin{array}{c}\text { ALBUMIN } \\
\mathrm{g} / \mathrm{dL}\end{array}$ & $\begin{array}{c}\text { TOTAL PROTEIN } \\
\mathrm{g} / \mathrm{dL}\end{array}$ & $\begin{array}{c}\text { BILIRUBIN } \\
\mathrm{mg} / \mathrm{dL}\end{array}$ & $\begin{array}{c}\text { BUN } \\
\mathrm{mg} / \mathrm{dL}\end{array}$ & $\begin{array}{c}\text { CREATININE } \\
\mathrm{mg} / \mathrm{dL}\end{array}$ & \begin{tabular}{|c}
$\begin{array}{c}\text { CHOLESTEROL } \\
\mathrm{mg} / \mathrm{dL}\end{array}$ \\
\end{tabular} & $\begin{array}{c}\text { GLUCOSE } \\
\mathrm{mg} / \mathrm{dL}\end{array}$ & $\begin{array}{c}\text { CALCIUM } \\
\mathrm{mg} / \mathrm{dL}\end{array}$ & \begin{tabular}{|c}
$\begin{array}{c}\text { PHOSPHORUS } \\
\mathrm{mg} / \mathrm{dL}\end{array}$ \\
\end{tabular} & $\begin{array}{c}\text { BICARBONATE } \\
\mathrm{mEq} / \mathrm{L}\end{array}$ \\
\hline \multicolumn{15}{|l|}{ Vehicle } \\
\hline mean & 95.6 & 30.4 & 79.8 & 256.8 & 3.3 & 5.7 & 0.2 & 39.4 & $<0.3$ & 95.8 & 207.2 & 10.4 & 8.9 & 25.2 \\
\hline SD & 12.0 & 8.0 & 65.1 & 302.2 & 0.5 & 0.7 & 0.1 & 6.7 & & 20.0 & 25.0 & 1.0 & 0.9 & 3.6 \\
\hline \multicolumn{15}{|l|}{ siRNA PNP, $11 \mathrm{mg} / \mathrm{kg}$} \\
\hline mean & 55.4 & 18.8 & 56.4 & 200.6 & 2.8 & 5.0 & $<0.3$ & 33.2 & $<0.3$ & 85.4 & 243.4 & 10.1 & 8.2 & 25.4 \\
\hline SD & 8.4 & 1.8 & 9.2 & 84.6 & 0.1 & 0.1 & & 4.1 & & 9.9 & 19.0 & 0.2 & 0.9 & 2.2 \\
\hline Reference range & $47-85$ & & & & & & & & & & & & & \\
\hline \begin{tabular}{|l|} 
One-way ANOVA with Dunnett's \\
multiple comparison test
\end{tabular} & $* *$ & NS & NS & NS & NS & NS & NS & NS & NS & NS & NS & NS & NS & NS \\
\hline
\end{tabular}


Supplementary Table 4. Cytokine measurements 2 and $6 \mathrm{~h}$ following treatment with either vehicle (10\% sucrose) or siRNA PNPs administered a single dose of $3 \mathrm{mg} / \mathrm{kg}$.

\begin{tabular}{|c|c|c|c|c|c|c|c|c|}
\hline Treatment & $\begin{array}{c}\mathrm{mIL} 1 \alpha \\
\mathrm{pg} / \mathrm{mL}\end{array}$ & $\begin{array}{c}\mathrm{mlL} 1 \beta \\
\mathrm{pg} / \mathrm{mL}\end{array}$ & $\begin{array}{l}\mathrm{mIL6} \\
\mathrm{pg} / \mathrm{mL}\end{array}$ & $\begin{array}{c}\mathrm{mlL} 10 \\
\mathrm{pg} / \mathrm{mL}\end{array}$ & $\begin{array}{c}\mathrm{mIL} 12 \mathrm{p} 70 \\
\mathrm{pg} / \mathrm{mL}\end{array}$ & $\begin{array}{c}\mathrm{mTNF} \alpha \\
\mathrm{pg} / \mathrm{mL}\end{array}$ & $\begin{array}{l}\mathrm{mKC} \\
\mathrm{pg} / \mathrm{mL}\end{array}$ & $\begin{array}{c}\mathrm{mIFN} \gamma \\
\mathrm{pg} / \mathrm{mL}\end{array}$ \\
\hline \multicolumn{9}{|l|}{ Vehicle 2 hrs } \\
\hline mean & 0 & 0 & 0 & 0 & 0 & 0 & 8 & 0 \\
\hline SD & 0 & 0 & 0 & 0 & 0 & 0 & 9 & 0 \\
\hline \multicolumn{9}{|l|}{ Vehicle 6 hrs } \\
\hline mean & 0 & 0 & 0 & 0 & 0 & 0 & 2 & 47 \\
\hline SD & 0 & 0 & 0 & 0 & 0 & 0 & 2 & 106 \\
\hline \multicolumn{9}{|l|}{ LPS 2 hrs } \\
\hline mean & 0 & 0 & 112728 & 25 & 2 & 140 & 473 & 6 \\
\hline SD & 0 & 0 & 144593 & 13 & 1 & 67 & 235 & 2 \\
\hline $\begin{array}{l}\text { One-way ANOVA with Dunnett's } \\
\text { multiple comparison test }\end{array}$ & NS & NS & $* *$ & $* * *$ & $* * *$ & $* * *$ & $* *$ & NS \\
\hline \multicolumn{9}{|l|}{ LPS 6 hrs } \\
\hline mean & 0 & 0 & 1102 & 4 & 1 & 27 & 215 & 3438 \\
\hline SD & 0 & 0 & 493 & 1 & 0 & 10 & 63 & 2535 \\
\hline $\begin{array}{l}\text { One-way ANOVA with Dunnett's } \\
\text { multiple comparison test }\end{array}$ & NS & NS & NS & NS & NS & NS & NS & $* * *$ \\
\hline \multicolumn{9}{|l|}{ siRNA PNP 2 hrs } \\
\hline mean & 0 & 0 & 39 & 0 & 0 & 13 & 53 & 0 \\
\hline SD & 0 & 0 & 32 & 0 & 0 & 9 & 26 & 0 \\
\hline $\begin{array}{l}\text { One-way ANOVA with Dunnett's } \\
\text { multiple comparison test }\end{array}$ & NS & NS & NS & NS & NS & NS & NS & NS \\
\hline \multicolumn{9}{|l|}{ siRNA PNP 6 hrs } \\
\hline mean & 0 & 0 & 0 & 0 & 0 & 0 & 0 & 0 \\
\hline SD & 0 & 0 & 0 & 0 & 0 & 0 & 0 & 0 \\
\hline $\begin{array}{l}\text { One-way ANOVA with Dunnett's } \\
\text { multiple comparison test }\end{array}$ & NS & NS & NS & NS & NS & NS & NS & NS \\
\hline
\end{tabular}

